\title{
Nuchal Translucency in Early Pregnancy
}

\author{
Dr.R.Padma devi ${ }^{1}$, Dr.N.Geetha ${ }^{2}$ \\ ${ }^{1,2}$ (Department Of Obstetrics \& Gynaecology, GMKMCH/TN Dr MGR University, India)
}

\begin{abstract}
The aim of the study is to detect whether nuchal translucency can be used as an early screening tool to identify fetal abnormalities so that termination can be done at early pregnancy itself in case of anomalous fetus.

Methods: Prospective study conducted among randomly selected pregnant women with singleton pregnancy between 10-14 weeks of gestation, with regular menstrual cycle and with reliable LMP details attending the antenatal outpatient department. $1^{\text {st }}$ trimester scan with $N T$ measurement, $2^{\text {nd }}$ trimester target scan, fetal Echocariography were done. If major anomalies were present, termination was done. Others were followed till delivery and the baby was examined after delivery.

Results: In this study, 96\% pregnant women had normal NT value (<95\%centile). None of them had major anomalies. $P$ value is 0.017. Fetal anomalies are significantly related to increased value of nuchal translucency. Conclusion: NT is a non-invasive reliable screening tool available in $1^{\text {st }}$ trimester. NT measurement combined with other sonographic markers would be ideal consideration for $1^{\text {st }}$ trimester termination.
\end{abstract}

Keywords : Nuchal translucency, screening, fetal anomaly, sonographic marker, termination

\section{Introduction}

One of the important cause of perinatal death and childhood handicap is chromosomal abnormalities. Prenatal screening has been increasing over the past 20years for Down's syndrome and other aneuploidies. The choice of definitive diagnosis of aneuploidy was limited to amniocentesis which was performed at 15 weeks of gestation at the earliest and prenatal diagnosis was made in the second trimester. Moreover, amniocentesis was invasive procedure and pregnancy loss rate is 1 in $200^{1}$.First trimester screening includes nuchal translucency measurement, presence of nasal bone and frontomaxillary facial angle measurement sonographically, biochemical markers like PAPP-A and $\beta$-HCG, and Doppler study of tricuspid valve and ductus venosus ${ }^{2}$. With these screening facilities, prenatal screening has moved from $2^{\text {nd }}$ trimester to $1^{\text {st }}$ trimester screening.

\section{Method}

This prospective study was conducted at Coimbatore Medical College Hospital, Coimbatore. The study has been conducted on 50 pregnant women between 10-14 weeks of gestation with regular menstrual cycle, reliable LMP details, and with singleton pregnancy, attending the antenatal outpatient department.The selection of patients was random and everyone was counselled about the study. Detailed history was taken and any risk factor contributing to fetal abnormality was noted. Thorough clinical examination was done. Preliminary investigations were carried out at 10-14 weeks. USG done to measure nuchal translucency, fetal CRL, and any obvious structural anomaly in the fetus, uterine adnexa, cervix and internal os were noted.After the scan estimated risk of Down's syndrome or other fetal abnormalities are discussed with the pregnant women and her family. If she is at high risk, she will be counselled and adviced to wait for the target scan and fetal echocardiogram. If major anomalies are present, she will be adviced for termination of pregnancy. All women were followed up till delivery,adviced for institutional delivery and the baby was examined after delivery.

\section{Results}

In this study majority of the women were between $21-25$ years(50\%),34\% were between 26 30 years, $56 \%$ were primigravida, $44 \%$ were multigravida. Most of the women were screened between 12-13 weeks. $22 \%$ of the pregnant women had significant past obstetric complications like $1^{\text {st }}$ and $2^{\text {nd }}$ trimester loss, IUD, preterm delivery, IUGR, GDM, GHT, anomalous baby. Rest of them had no previous obstetric complications. $96 \%$ of pregnant women had NT less than $95^{\text {th }}$ centile. $4 \%$ of pregnant women had NT more than $95^{\text {th }}$ centile. 48 pregnant women in our study $(96 \%)$ had normal nuchal translucency value $(<95 \%$ centile). 2 pregnant women had increased nuchal translucency value( $>95 \%$ centile). All the pregnant women in the study were followed with anomaly scan and fetal echocardiography. Among the 2 pregnant women with increased NT, one of the baby had isolated cardiac defect(ASD), and the pregnancy was continued. The other pregnant woman had no anomaly in the follow up scan and the pregnancy was continued.Among the 48 pregnant women with normal NT, one of the baby had patent ductus arteriosus. Rest of the babies were normal. Increased NT identifies the group at risk not only for chromosomal abnormalities but also for major cardiac defects. The 
sensitivity of fetal NT $>95^{\text {th }}$ centile used for screening anomalies is $50 \%$, specificity is $97.92 \%$, and the $\mathrm{P}$ value is 0.017. fetal anomalies are significantly related to nuchal translucency.

\section{Discussion}

1) Nuchal translucency must be measured at 10-14 weeks of gestation when CRL corresponds to 38$84 \mathrm{~mm}$ with fetus in neutral position and viewed in mid saggital section ${ }^{3}$. The maximum thickness of subcutaneous translucency between the skin and the soft tissue overlying the cervical spine is measured as Nuchal translucency. Multiple measurements are taken and the maximum reading is taken to be more accurate.

2) The fetal lymphatic system is developing between 10-14weeks of gestation and the peripheral resistance of the placenta is also high, so the excess fluid accumulates behind the neck leading to increased $\mathrm{NT}^{4}$. After 14 weeks of gestation, the lymphatic system is well developed to drain away the excess fluid to placental circulation. After this period fetal abnormalities causing fluid accumulation will be corrected by itself and goes undetected by measuring NT.

3) Nuchal translucency rises with increasing gestational age. The cut off on this progressive rise is used as $95^{\text {th }} \%$ centile corresponding to gestational age, which results in the more sensitive and specific indicator for detection of anomalous fetus ${ }^{5}$. NT increases with increasing gestational age and with increasing crown rump length. While NT measurement, it is important to correlate with gestational age, to determine whether given NT thickness is increased.

4) Increasd NT is associated with chromosomal abnormalities. Even chromosomally normal foetuses with cardiac defects, pulmonary defect, abdominal wall defect, central nervous system defect, neuromuscular defect, GIT defects, GUT defects, skeletal and metabolic defects,genetic syndrome will be associated with increased $\mathrm{NT}^{6}$.

5) The ideal test for all pregnant mothers should be non-invasive, less expensive and should give reliable information at the earliest regarding the fetal anomaly so that the affected women would be offered an early option of termination of pregnancy. All the above conditions of an ideal screening test is fulfilled by Nuchal Translucency.

V. Table

\begin{tabular}{|l|l|l|}
\hline Nuchal Translucency & Number of pregnant women & Percentile \\
\hline$\left\langle\mathbf{9 5}^{\text {th }}\right.$ centile & 48 & 96 \\
\hline$>\mathbf{9 5}^{\text {th }}$ centile & 2 & 4 \\
\hline
\end{tabular}

\section{Figure}

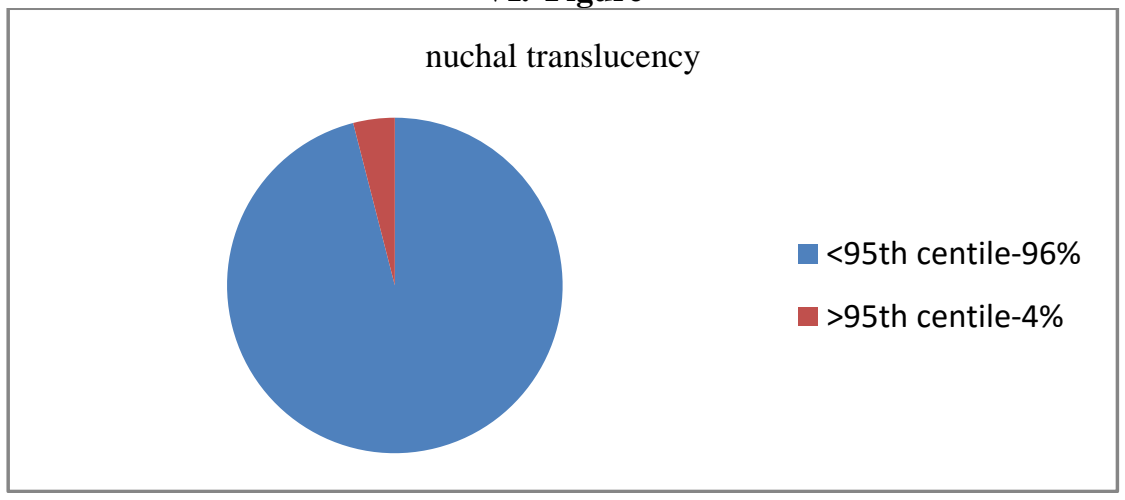

\section{Conclussion}

1) NT measurement is a non-invasive reliable screening tool available in the first trimester to determine the fetus at risk for fetal aneuploidy, cardiac defects, structural defects, genetic syndromes. 2) Apart from nuchal translucency, $1^{\text {st }}$ trimester USG is also used to confirm fetal viability, accurate dating of pregnancy, early diagnosis of multiple pregnancy and detection of major structural abnormalities, condition of the uterus, adnexa and cervical length. 3)Measurement of normal NT reduces the number of invasive procedures like CVS, amniocentesis and cordocentesis. 4) Measurement of increased NT provides women with affected foetuses an early termination option. 5) NT measurements in the $1^{\text {st }}$ trimester has pushed the prenatal screening of fetal anomalies from $2^{\text {nd }}$ trimester to the $1^{\text {st }}$ trimester. 


\section{References}

[1]. Malone FD, Berkowitz RL, Canick JA, D’Alton ME, First trimester screening for aneuploidy: research or of standard of care. Am J Obstet Gynecol 2000; 182: 490-6.

[2]. Prathima Radhakrishnan, Nicolaides KH, The 11-14 weeks scan- Fetal nuchal translucency. J Perinatol 2003; 5: 167-176.

[3]. Whitlow BJ, Economides DL. The optimal gestational age to examine fetal anatomy and measure nuchal translucency in the first trimester. Ultrasound gynaecol 1998; 11 : 258.

[4]. Von Kaisenberg CS, Nicolaides KH, Brand - Saberi B, Lymphatic Vessel hypoplasia in fetuses with Turner Syndrome. Hum Reprod 1999; 14: 823-6.

[5]. Nicolaides KH, Brijot ML, Snijders RJM, Fetal nuchal translucency; ultrasound screening for fetal trisomy in the first trimester of pregnancy. Br. J of Obstet Gynaecol 1994; 101: 782-86.

[6]. Hyett J, Moscoso G, Nicolaides KH. Abnormalities of the heart and great arteries in first trimester chromosomally abnormal fetuses. Am J. Med genet 1997; 69: 207-16. 